\title{
Efecto del Gas de Protección, Ángulos de Trabajo y Desplazamiento sobre las Características de Juntas de Filete Obtenidas por GMAW en un Acero Microaleado de Alta Resistencia
}

\author{
César Marconi ${ }^{1}$, María José Castillo ${ }^{1,2}$, Mabel Ramini ${ }^{1}$, Hernán Svoboda ${ }^{1,3,4}$ \\ 1 Universidad Tecnológica Nacional, Facultad Regional San Nicolás, Centro de Desarrollo y Tecnología de Materiales - \\ DEYTEMA, Provincia de Buenos Aires, Argentina. \\ 2 Universidad Nacional del Noroeste de la Provincia de Buenos Aires - UNNOBA, Escuela de Tecnología, Junín, Provincia de \\ Buenos Aires, Argentina. \\ 3 Universidad de Buenos Aires, Facultad de Ingeniería, Instituto de Tecnologías y Ciencias de la Ingeniería - INTECIN, \\ Laboratorio de Materiales y Estructuras, Grupo de Tecnología de la soldadura, Ciudad de Buenos Aires, Argentina. \\ 4 Consejo Nacional de Investigaciones Científicas y Técnicas, Ciudad de Buenos Aires, Argentina.
}

Recibido: 04 Marzo, 2016

Aprobado: 25 Jul., 2016

E-mail: cmarconi@frsn.utn.edu.ar(CM)
Resumen: La junta de filete es una de las más utilizadas en diversas industrias, en particular en la de maquinaria agrícola. Sin embargo, muchas veces, la complejidad de las piezas a unir dificulta la accesibilidad del soldador en este tipo de juntas, siendo los ángulos de trabajo y desplazamiento un aspecto importante a evaluar. Además, en el proceso GMAW el empleo de la mezcla Ar- $20 \% \mathrm{CO}_{2}$ como gas de protección se ha incrementado en detrimento del $100 \% \mathrm{CO}_{2}$, requiriendo en general un ajuste de los parámetros eléctricos. Por otro lado, la incorporación de nuevos materiales de mayor resistencia también requiere la optimización del procedimiento de soldadura. El objetivo de este estudio fue evaluar el efecto de dos gases de protección ( $\mathrm{Ar}-20 \% \mathrm{CO}_{2}$ y $\left.100 \% \mathrm{CO}_{2}\right)$, de tres ángulos de trabajo $\left(30,45\right.$ y $60^{\circ}$ ) y dos de desplazamiento $\left(45^{\circ}\right.$ por empuje y $60^{\circ}$ por arrastre) sobre los aspectos dimensionales del cordón y la resistencia mecánica de la junta de filete de un acero microaleado de alta resistencia, soldado mediante GMAW. Los mayores niveles de penetración correspondieron a las probetas soldadas por arrastre y con $\mathrm{CO}_{2}$. El sobre espesor fue menor para la condición por empuje y la mezcla de gases. La microdureza del metal de soldadura, para similar ángulo de trabajo, resultó mayor para el gas mezcla en todos los casos. Se desarrollaron índices de aceptabilidad que ponderan los aspectos geométricos y se los relacionaron con las propiedades mecánicas obtenidas sobre las juntas, mostrando una buena correlación.

Palabras clave: GMAW; Junta de filete; Gas de protección; Ángulo de trabajo y desplazamiento; Acero microaleado.

\section{Effect of Shielding Gas, Work and Travel Angles on Fillet Weld Characteristics Obtained by GMAW on a High Resistance Microalloyed Steel}

\begin{abstract}
The fillet joint is one of the most used in several industries, particularly in the agricultural machinery. However, many times, the complexity of the parts to be joined difficult welder accessibility, therefore working and travel angles are important variables to analyze. Furthermore, in GMAW process, employing shielding gas mixture $\mathrm{Ar}-20 \% \mathrm{CO}_{2}$ has increased at expense of $100 \% \mathrm{CO}_{2}$, requiring in general electrical parameters adjustment. In addition, incorporating new higher strength materials also requires optimizing the welding procedure. The aim of this study was to evaluate the effect of two shielding gases $\left(\mathrm{Ar}-20 \% \mathrm{CO}_{2}\right.$ and $100 \% \mathrm{CO}_{2}$ ), three work angles $\left(30,45\right.$ and $60^{\circ}$ ) and two travel angles ( $45^{\circ}$ for push welding and $60^{\circ}$ for drag welding) on the dimensional aspects and mechanical properties of joints, obtained by GMAW on a microalloyed high strength steel. The highest levels of penetration corresponded to the samples welded by drag welding and $\mathrm{CO}_{2}$. The reinforcement was lower for pushing welding condition and mixture shield gases. The weld metal microhardness, to similar work angles, was higher for the gas mixture in all cases. Performance index were developed to consider the several geometrical aspect of the joints. They were correlated whit mechanical properties showing a good agreement.
\end{abstract}

Key-words: GMAW; Fillet joint; Shielded gas; Work and travel angles; Microalloyed steels. 


\section{Introducción}

En la actualidad, uno de los procesos de fabricación más empleados en estructuras metálicas es la soldadura [1]. Sin embargo, existen antecedentes que demuestran que en las empresas del sector metalmecánico y afines existe un fuerte desconocimiento sobre distintos aspectos relacionados con la soldadura, así como poca evaluación e investigación de las tecnologías y procedimientos de soldadura [2]. En este sentido, las instituciones educativas en las áreas de ingeniería, afines con la tecnología de soldadura juegan un rol de relevancia en el desarrollo de metodologías que faciliten la mejora y aseguramiento de la calidad de los productos soldados.

La junta de filete es una de las más utilizadas en diversas industrias, en particular en la de maquinaria agrícola debido a que no requiere demasiada preparación. Además, en ocasiones, el depósito puede realizarse en una pasada y, en consecuencia, resulta de bajo costo [3]. En muchas circunstancias la complejidad de las piezas a unir dificulta la accesibilidad del soldador en este tipo de juntas, por lo que las condiciones de proceso no son las habituales. En este sentido, el ángulo de trabajo y desplazamiento, tanto por empuje como por arrastre, juegan un rol importante a la hora de la selección de los mismos según las posibilidades de acceso a la junta. Es sabido que el ángulo de trabajo recomendado es el de $45^{\circ}$ y el de desplazamiento de 60 a $75^{\circ}$ por arrastre, lo que permite una buena penetración y calidad del cordón [4-6]. Sin embargo, debido a la falta de acceso a la junta no siempre es posible emplear esa condición ideal. La complejidad de las variables puestas en juego y el modo de interacción entre cada una de ellas llevan muchas veces a una solución de compromiso. En tal sentido, analizar el efecto de estos ángulos sobre las características dimensionales de los cordones de soldadura obtenidos en junta de filete para aplicaciones en la industria de maquinaria agrícola es un aspecto de interés y sobre el que no se encuentra demasiada información disponible. Asimismo, determinar un índice de aceptabilidad que permita comparar cada una de las configuraciones es importante para evaluar la calidad geométrica y morfológica de la junta soldada.

Por otro lado, en los últimos años en el mercado local argentino vinculado a la maquinaria agrícola, en el proceso GMAW (Gas Metal Arc Welding) el empleo de una mezcla de gas protector constituido por 80\% Ar y

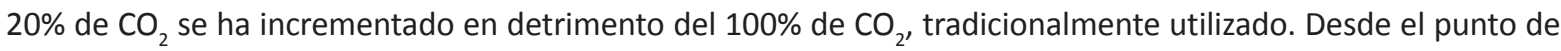
vista productivo, la utilización de esta mezcla de gases permite incrementar levemente los parámetros eléctricos (tensión y corriente) de soldadura y, de este modo, la velocidad de avance. Esto se debe fundamentalmente a la diferencia de conductividad existente entre el $\mathrm{CO}_{2}$ y el $\mathrm{Ar}$, donde este último al poseer una baja conductividad requiere de incrementos en el voltaje de arco con el objetivo de aumentar el calor aportado [4]. Además, el tipo de gas protector afecta la forma de la pileta líquida y las propiedades mecánicas [7]. Asimismo, afecta el modo de transferencia pudiendo lograr un modo tipo spray que disminuye las salpicaduras y provee un excelente aspecto superficial [8-10]. Sin embargo, esta modificación requiere de la puesta a punto del procedimiento de soldadura para no afectar la calidad de la junta soldada en cuanto a niveles de defectos y penetración.

Por último, la incorporación de nuevos materiales de mayor resistencia es un requerimiento actual a fin de disminuir el peso de los equipos [11]. Sin embargo, esto también requiere de la optimización del procedimiento de soldadura. En la selección del consumible para realizar una junta soldada existen diferentes opciones que el especialista en soldadura debe analizar. Una teoría muy difundida es la que propone que el metal de soldadura debe tener una resistencia igual o mayor al metal base, de manera que cuando se ensaye la junta a la tracción la fractura ocurra fuera de la soldadura [12].

El objetivo de este estudio fue evaluar el efecto del gas de protección, y de los ángulos de trabajo y desplazamiento sobre los aspectos dimensionales del cordón soldado y la resistencia mecánica en la junta de filete de un acero microaleado de alta resistencia para aplicaciones en la industria de maquinaria agrícola. Asimismo, se buscó determinar un índice de aceptabilidad que permita comparar cada una de las configuraciones de soldadura con el fin de evaluar la calidad geométrica y morfológica de la junta de filete.

\section{Materiales y Métodos}

A fin de alcanzar los objetivos propuestos se soldaron probetas mediante el proceso GMAW empleando una junta tipo filete bajo distintas configuraciones operativas. 
Efecto del Gas de Protección, Ángulos de Trabajo y Desplazamiento sobre las Características de Juntas de Filete Obtenidas por GMAW en un Acero Microaleado de Alta Resistencia

\subsection{Material base}

Como metal base se utilizó un acero microaleado de alta resistencia (HSLA equivalente al grado 060 XLF de la Norma SAE J1392) de $6 \mathrm{~mm}$ de espesor, sobre el cual se determinó la composición química mediante espectrometría de emisión óptica (OES) y se realizó la caracterización microestructural mediante microscopia óptica (LM) y electrónica de barrido (SEM). Asimismo, se determinaron las propiedades mecánicas mediante mediciones de microdureza Vickers (HV1 kg) y ensayo de tracción uniaxial, según la Norma ASTM E8-04 [13].

\subsection{Soldadura}

Para la soldadura de las probetas se realizaron cupones de junta soldada, según la Especificación AWS D14.3/D14.3M:2010 [14]. Los mismos se soldaron utilizando una fuente semiautomática multipropósito Aristo U500 de ESAB en forma mecanizada con un dispositivo de avance Miggytrac 1500 (Figura 1), se configuraron tres ángulos de trabajo $\left(30,45\right.$ y $\left.60^{\circ}\right)$, dos de desplazamiento ( $45^{\circ}$ por empuje y $60^{\circ}$ por arrastre) (Figura 2 ), y dos gases de protección ( $\mathrm{Ar}-20 \% \mathrm{CO}_{2}$ y $100 \% \mathrm{CO}_{2}$ ). La posición de soldadura utilizada fue horizontal, bajo mano. El caudal de gas empleado fue de $12 \mathrm{~L} / \mathrm{min}$ y la distancia pico pieza de $13 \mathrm{~mm}$. Como metal de aporte se empleó alambre macizo ER80S-D2 de 1,2 mm de diámetro. Sobre este material se realizó un cupón de aporte puro para la determinación de la composición química del depósito según la Especificación AWS A5.28/A5.28M:2005 [15].

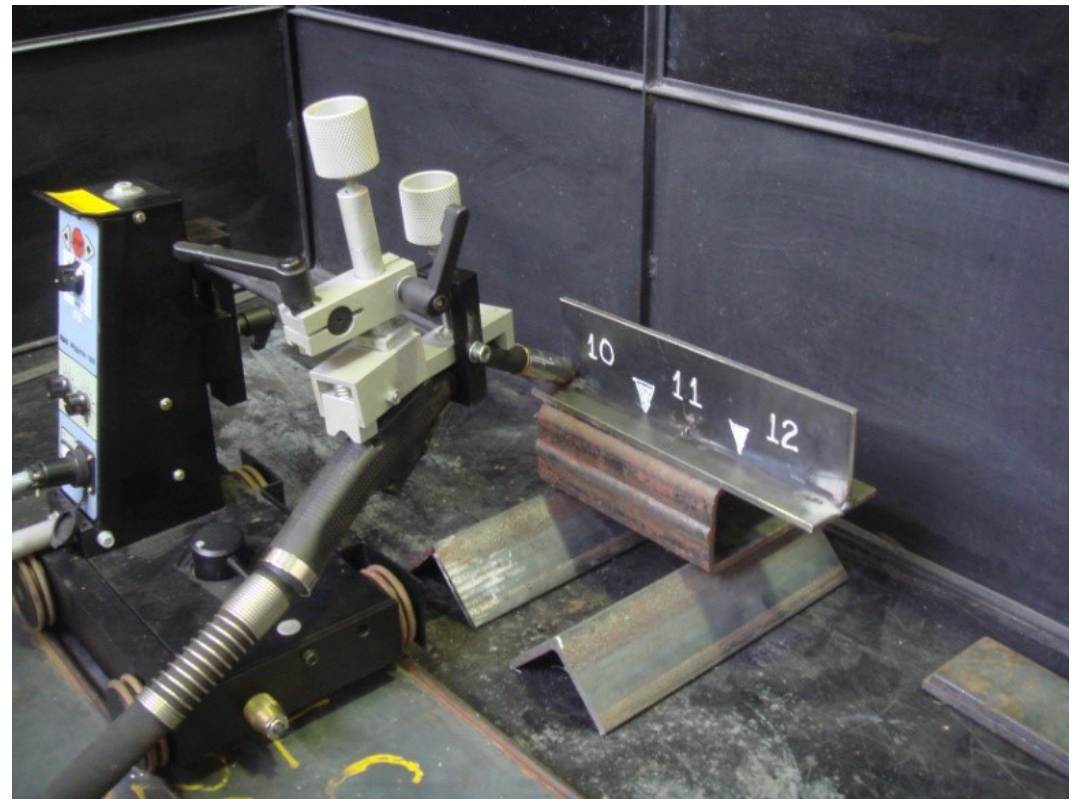

Figura 1. Montaje experimental empleado.

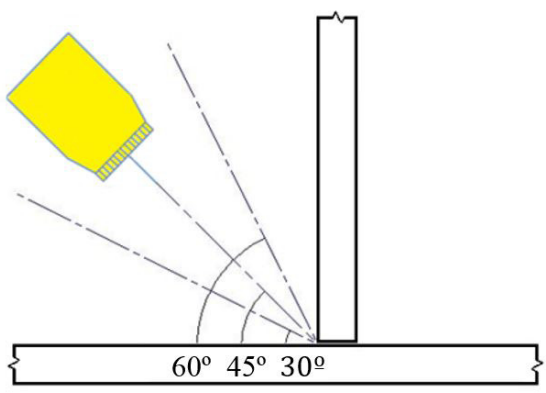

a

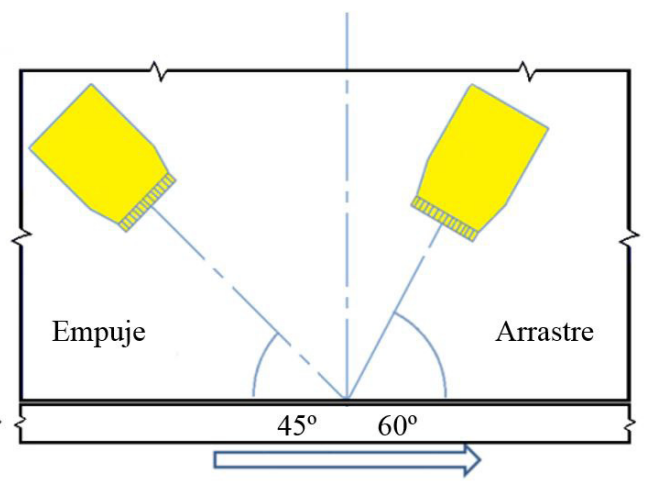

b

Figura 2. Esquema de las configuraciones del ángulo de trabajo (a) y desplazamiento (b). 
Con el fin de poder comparar los resultados se mantuvo el nivel de calor aportado ( $\mathrm{HI}$ : heat input) para cada cupón de soldadura. Para ello se optimizaron los parámetros eléctricos de trabajo y se modificó la velocidad de avance en cada caso. La identificación de cada cupón, los parámetros de soldadura utilizados y el HI resultante se muestran en la Tabla 1; junto con las distintas configuraciones de ángulos y gases analizados. Se mantuvieron constantes la velocidad de alimentación del alambre y el voltaje y se midió la intensidad de corriente y la tensión medias durante la ejecución de los cordones. El calor aportado se determinó de acuerdo a la Ecuación 1:

$$
\mathrm{HI}=\frac{\mathrm{V} \times \mathrm{I}}{\mathrm{Vs}}
$$

Tabla 1. Parámetros de soldadura e identificación de los cupones.

\begin{tabular}{|c|c|c|c|c|c|c|c|c|}
\hline \multirow[b]{2}{*}{ Probeta } & Va & $\mathbf{V}$ & I & Vs & $\mathrm{HI}$ & \multirow{2}{*}{$\begin{array}{c}\text { Ángulo } \\
\text { Desplazamiento }\end{array}$} & \multirow{2}{*}{$\begin{array}{l}\text { Ángulo } \\
\text { Trabajo }\end{array}$} & \multirow[b]{2}{*}{ Gas Protector } \\
\hline & $\begin{array}{c}(\mathrm{m} / \\
\mathrm{min})\end{array}$ & (V) & (A) & $(\mathrm{mm} / \mathrm{s})$ & $(\mathrm{kJ} / \mathrm{mm})$ & & & \\
\hline CA 30 & 5,5 & 23 & 176 & 3,6 & 1,1 & Arrastre, $60^{\circ}$ & $30^{\circ}$ & $100 \% \mathrm{CO}_{2}$ \\
\hline CA 45 & 5,5 & 23 & 178 & 3,6 & 1,1 & Arrastre, $60^{\circ}$ & $45^{\circ}$ & $100 \% \mathrm{CO}_{2}$ \\
\hline CA 60 & 5,5 & 23 & 184 & 4,5 & 0,9 & Arrastre, $60^{\circ}$ & $60^{\circ}$ & $100 \% \mathrm{CO}_{2}$ \\
\hline CE 30 & 5,5 & 23 & 190 & 4,4 & 1,0 & Empuje, $45^{\circ}$ & $30^{\circ}$ & $100 \% \mathrm{CO}_{2}$ \\
\hline CE 45 & 5,5 & 23 & 171 & 4,5 & 0,9 & Empuje, $45^{\circ}$ & $45^{\circ}$ & $100 \% \mathrm{CO}_{2}$ \\
\hline CE 60 & 5,5 & 23 & 169 & 4,2 & 0,9 & Empuje, $45^{\circ}$ & $60^{\circ}$ & $100 \% \mathrm{CO}_{2}$ \\
\hline ME 30 & 5,5 & 23 & 195 & 4,7 & 1,0 & Empuje, $45^{\circ}$ & $30^{\circ}$ & $80 \% \mathrm{Ar}-\mathrm{CO}_{2}$ \\
\hline ME 45 & 5,5 & 23 & 182 & 4,2 & 1,0 & Empuje, $45^{\circ}$ & $45^{\circ}$ & $80 \% \mathrm{Ar}-\mathrm{CO}_{2}$ \\
\hline ME 60 & 5,5 & 23 & 171 & 3,4 & 1,1 & Empuje, $45^{\circ}$ & $60^{\circ}$ & $80 \% \mathrm{Ar}-\mathrm{CO}_{2}$ \\
\hline MA 30 & 5,5 & 23 & 203 & 4,1 & 1,1 & Arrastre, $60^{\circ}$ & $30^{\circ}$ & $80 \% \mathrm{Ar}-\mathrm{CO}_{2}$ \\
\hline MA 45 & 5,5 & 23 & 190 & 4,3 & 1,0 & Arrastre, $60^{\circ}$ & $45^{\circ}$ & $80 \% \mathrm{Ar}-\mathrm{CO}_{2}$ \\
\hline MA 60 & 5,5 & 23 & 179 & 4,0 & 1,0 & Arrastre, $60^{\circ}$ & $60^{\circ}$ & $80 \% \mathrm{Ar}-\mathrm{CO}_{2}$ \\
\hline
\end{tabular}

Va: Velocidad de alimentación de alambre; V: Tensión; I: Corriente; Vs: Velocidad de soldadura; HI: calor aportado.

\subsection{Macrografía y análisis dimensional}

Sobre los cupones soldados se realizaron cortes transversales al cordón y se analizó la macroestructura. Todas las muestras se desbastaron con papeles abrasivos de carburo de silicio hasta grado 600 , el pulido final se realizó utilizando pasta diamantada de 6 y $1 \mu \mathrm{m}$. La macroestructura de soldadura se reveló con Nital $2 \%$ y se obtuvieron imágenes con lupa estereoscópica (LM) Olympus SX61. Las mediciones se realizaron empleando el software de análisis de imágenes libre ImageJ de acuerdo a los esquemas de la Figura 3 según la Especificación [14].

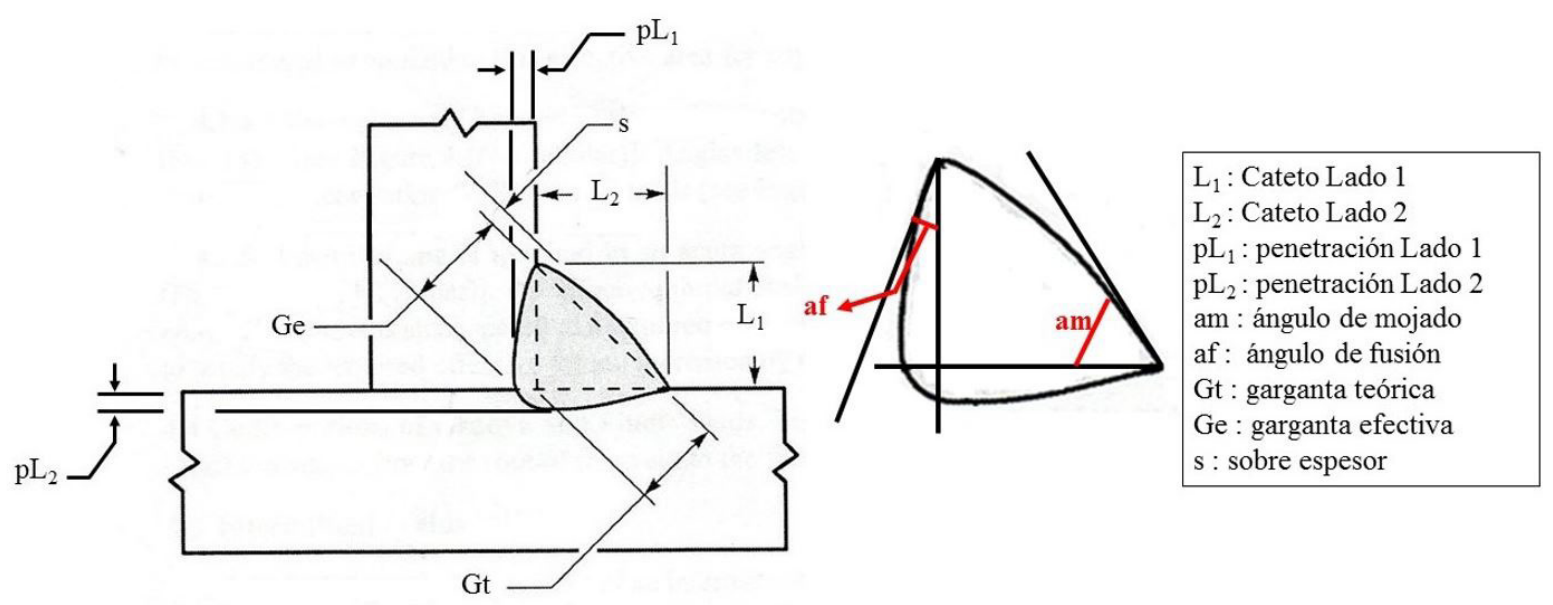

Figura 3. Esquema de las mediciones tomadas en las soldaduras de filete. 
Efecto del Gas de Protección, Ángulos de Trabajo y Desplazamiento sobre las Características de Juntas de Filete Obtenidas por GMAW en un Acero Microaleado de Alta Resistencia

El porcentaje de dilución se determinó de acuerdo a la Ecuación 2, midiendo las áreas de metal base fundido y aportada para cada configuración, según el esquema de la Figura 4.

$$
\% \mathrm{D}=\frac{\mathrm{A}}{\mathrm{A}+\mathrm{B}} \times 100
$$

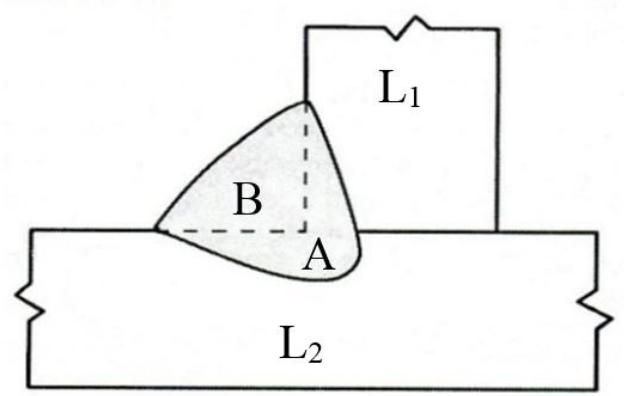

$\mathrm{L}_{1}$ : Lado 1

$\mathrm{L}_{2}$ : Lado 2

A: Área fundida del metal base

B: Área aportada por el consumible

Figura 4. Esquema de las áreas tomadas para el cálculo de la dilución.

\subsection{Ensayos mecánicos}

Se realizaron tres mediciones de microdureza Vickers (HV1 kg) en el metal de soldadura (MS) con un microdurómetro LECO LM 300 AT. Las improntas fueron realizadas sobre una línea paralela al borde superior de cada cordón, una en el centro y las otras dos cercanas a los lados, como se muestra en el esquema de la Figura 5. Los resultados obtenidos fueron promediados para cada condición de soldadura.

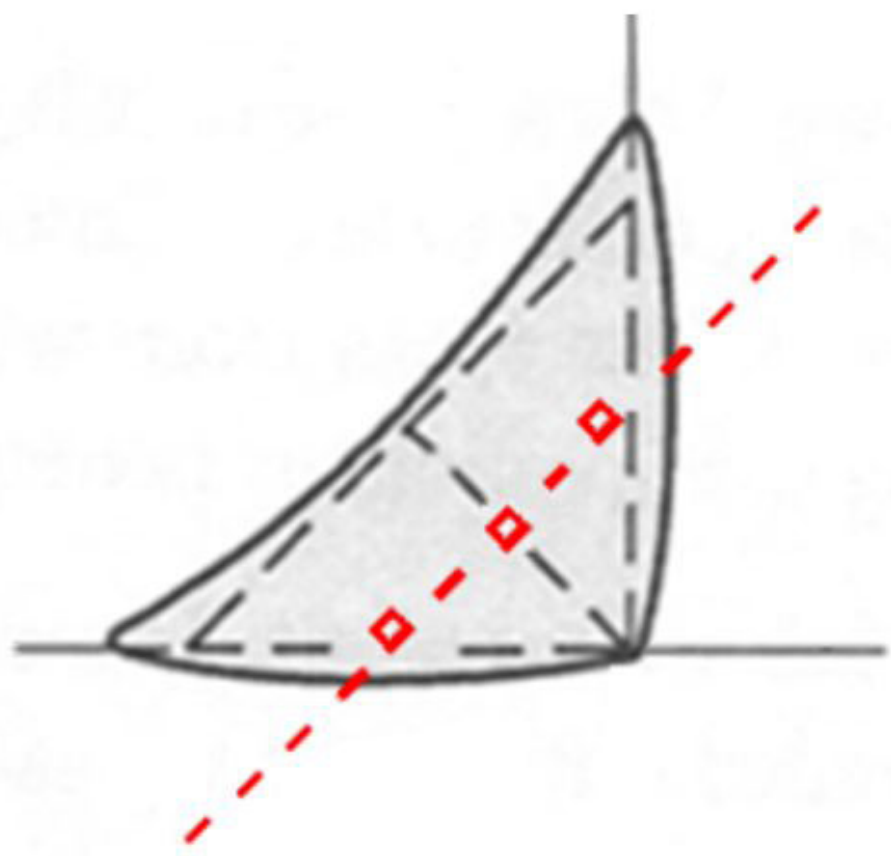

Figura 5. Esquema de ubicación de las improntas de microdureza en el MS.

Sobre dos probetas extraídas de cada cupón soldado se realizó el ensayo de rotura de acuerdo al esquema de la Figura 6. Las mismas fueron ensayadas, según la Especificación [14], con una Máquina Universal SSI-SATEC-BALDWIN de 30 t y los resultados fueron promediados para cada condición de soldadura. Se determinó la carga de rotura en cada caso, expresándola en carga $(\mathrm{N})$ por unidad de longitud de cordón ( $\mathrm{mm}$ ). 


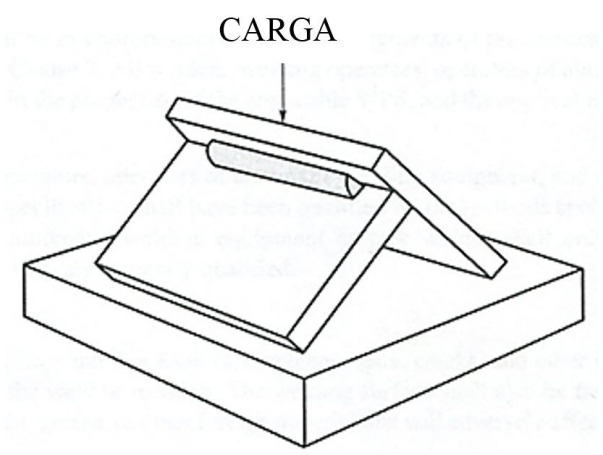

Figura 6. Esquema del ensayo de rotura de las juntas de filete.

\section{Resultados y Discusión}

\subsection{Caracterización del material base}

En la Tabla 2 se muestra la composición química del material empleado expresada en porcentaje en peso (wt\%), así como también el carbono equivalente calculado (Ceq IIW) [8].

Puede verse que corresponde a un acero de bajo contenido de carbono aleado al Mn y con la presencia de microaleantes ( $\mathrm{Ti} \mathrm{y} \mathrm{Nb}$ ). El Ceq es bajo, por lo que no se requiere precalentamiento.

En la Tabla 3 se muestran los resultados de las propiedades mecánicas del material base empleado.

Tabla 2. Composición química del metal base (wt\%).

\begin{tabular}{cccccccccccccccc}
\hline $\mathbf{C}$ & $\mathbf{M n}$ & $\mathbf{S i}$ & $\mathbf{S}$ & $\mathbf{P}$ & $\mathbf{C r}$ & $\mathbf{N i}$ & $\mathbf{M o}$ & $\mathbf{V}$ & $\mathbf{N b}$ & $\mathbf{A l}$ & $\mathbf{T i}$ & $\mathbf{C u}$ & $\mathbf{N}^{*}$ & $\mathbf{B}^{*}$ & $\mathbf{C e q}$ \\
0,082 & 1,223 & 0,132 & 0,006 & 0,019 & 0,029 & 0,028 & 0,010 & 0,003 & 0,043 & 0,058 & 0,037 & 0,04 & 40 & 2 & 0,32 \\
\hline
\end{tabular}

*Expresado en ppm.

Tabla 3. Propiedades mecánicas del metal base.

\begin{tabular}{cccc}
\hline HV1 & $\mathbf{R}(\mathbf{M P a})$ & $\mathbf{R f}(\mathbf{M P a})$ & $\mathbf{A}(\%)$ \\
197 & 610 & 516 & 27 \\
\hline
\end{tabular}

HV1: Microdureza Vickers (1 kg); R: resistencia a la tracción; Rf: tensión de fluencia; A: alargamiento.

Se observa que corresponde a un material de alta resistencia, superando los $600 \mathrm{MPa}$ de resistencia a la tracción.

En la Figura 7 se muestra la microestructura del acero base obtenida mediante LM y SEM.
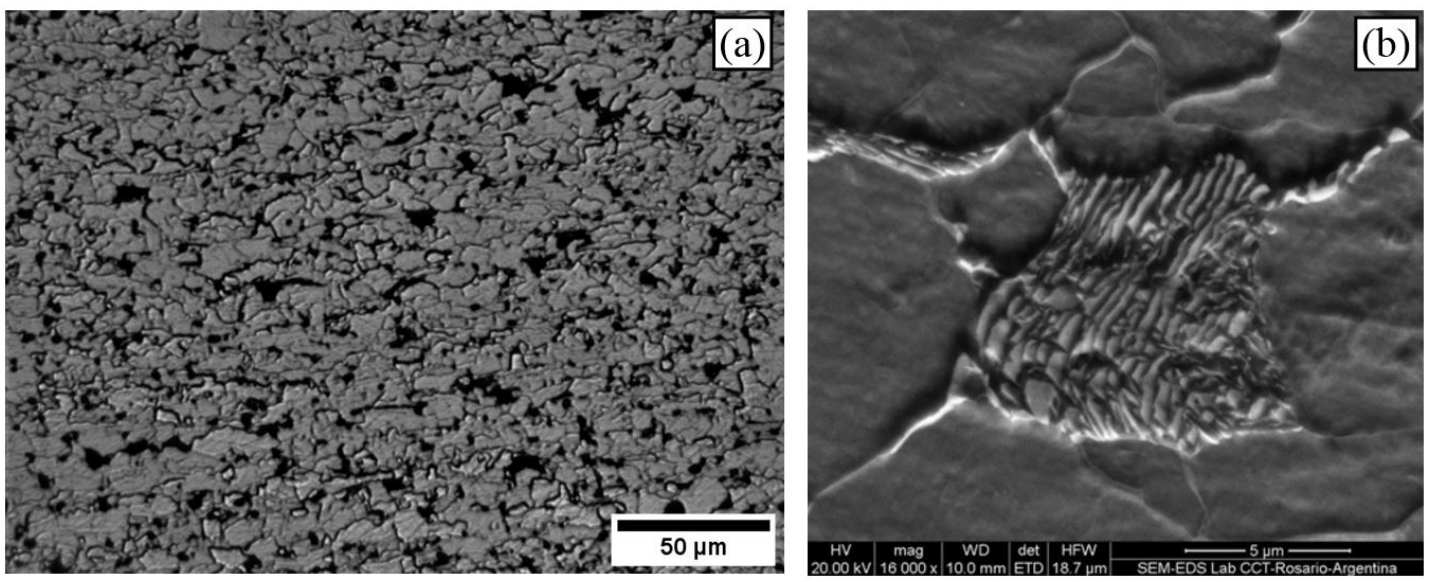

Figura 7. Microestructura del acero base, (a) microscopia óptica y (b) electrónica de barrido. 
Efecto del Gas de Protección, Ángulos de Trabajo y Desplazamiento sobre las Características de Juntas de Filete Obtenidas por GMAW en un Acero Microaleado de Alta Resistencia

Se distingue una estructura ferrítica con algo de perlita y tamaño de grano fino uniforme de 5,4 $\mu \mathrm{m}$ de diámetro en todo el espesor de la chapa.

\subsection{Inspección visual}

En cuanto a la operatividad de las diferentes configuraciones, el nivel de salpicaduras y la calidad superficial de los cordones fue muy bueno, sin encontrarse mayores dificultades ni defectos visibles. En la Figura 8 se muestran los aspectos superficiales de cada uno de los distintos cordones soldados. Se observa para las probetas soldadas por empuje la presencia de una pequeña capa de escoria adherida a ambos lados del cordón, tanto con $100 \%$ $\mathrm{CO}_{2}$ como con Ar-20\% $\mathrm{CO}_{2}$. Esto está asociado a la oxidación en la atmósfera del arco de elementos de aleación presentes como el Si o Mn y es habitualmente observado [16], siendo más significativo cuanto mayor es el potencial de oxidación del gas de protección empleado. La distancia entre aguas en todos los casos fue correcta y no se encontraron socavados ni solapes. Respecto a la eficiencia de la deposición no se perciben diferencias entre las diferentes configuraciones de ángulos y gases. Las probetas soldadas con $\mathrm{Ar}-20 \% \mathrm{CO}_{2}$ y por empuje presentan un cordón más plano y ancho con un mejor mojado, en cambio, en las soldadas con $100 \% \mathrm{CO}_{2}$ por arrastre se observaron en general cordones más convexos y estrechos con un peor mojado.
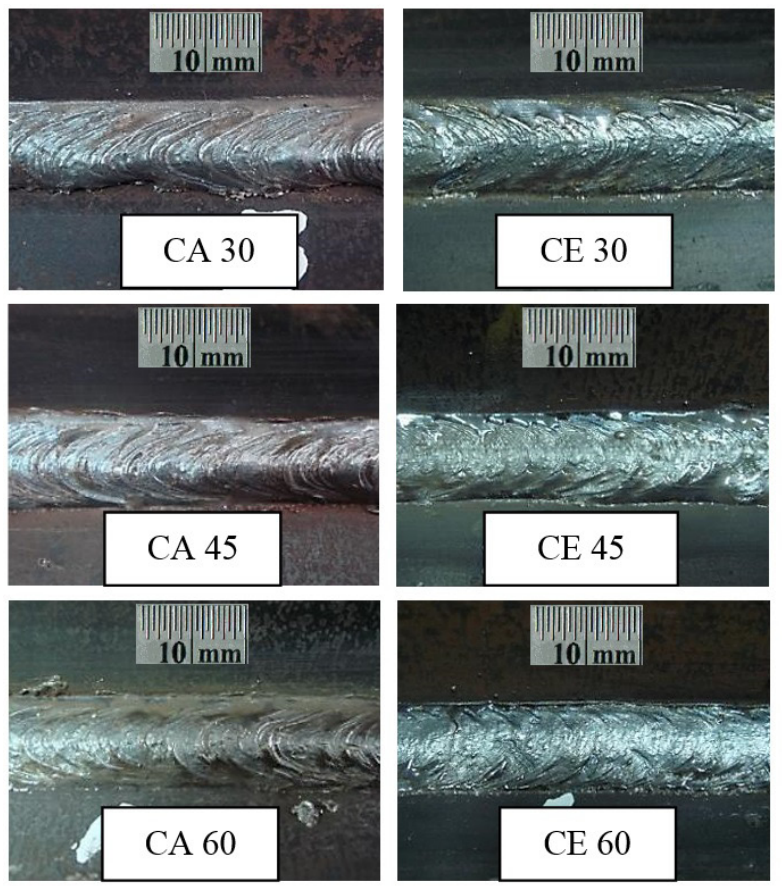
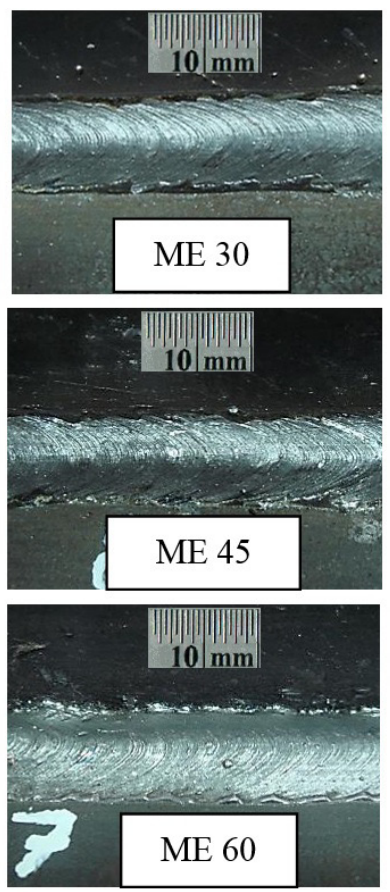
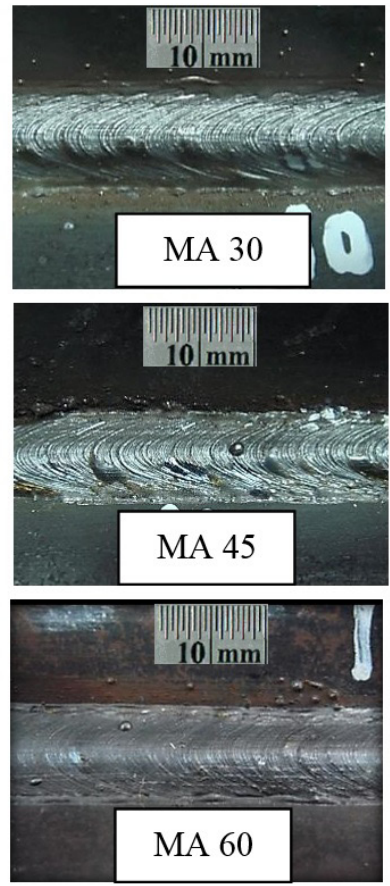

Figura 8. Aspecto superficial de los cordones de soldadura para las distintas configuraciones analizadas.

\subsection{Análisis dimensional}

La Figura 9 muestra las macrografías de las probetas soldadas. En las mismas se aprecian las distintas zonas de las juntas soldadas, la morfología de los cordones y los aspectos dimensionales.

En la Tabla 4 se muestran las mediciones realizadas de los parámetros geométricos de cada probeta.

Como se observa en los gráficos de la Figura 10, los mayores valores de garganta efectiva, correspondieron a las muestras soldadas con $100 \% \mathrm{CO}_{2}$ por arrastre, siendo 30 y $45^{\circ}$ los ángulos de trabajo óptimos en este sentido. Para la mezcla de gases Ar-20\% $\mathrm{CO}_{2}$ esta diferencia en el tamaño de la garganta efectiva entre la condición por arrastre y empuje no se notó teniendo la condición por empuje una $G_{e}$ levemente superior, aunque la penetración para esta mezcla de gases no satisface los requerimientos cuando los ángulos de trabajo se alejan del ideal a $45^{\circ}$. Esto será analizado más adelante en conjunto con otros aspectos como la dilución, los ángulos de fusión y mojado y la penetración en los lados. Aquellos casos donde la garganta efectiva no supero a la teórica son remarcados en 


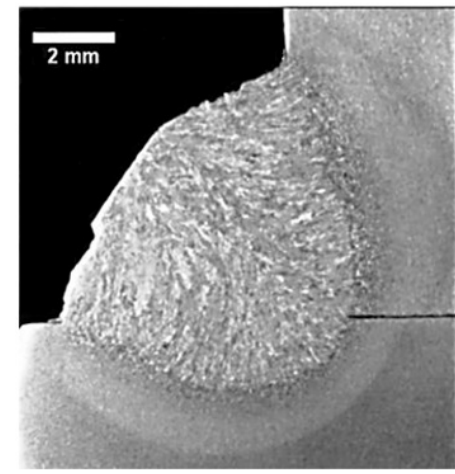

CA 30

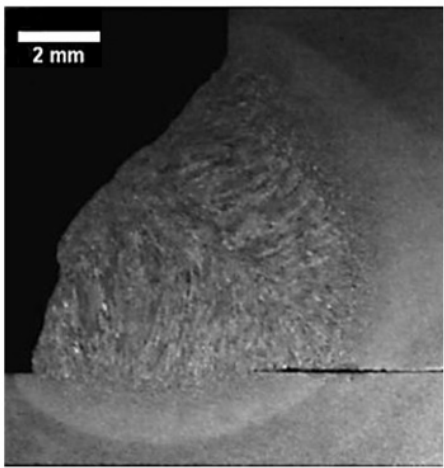

CE 30

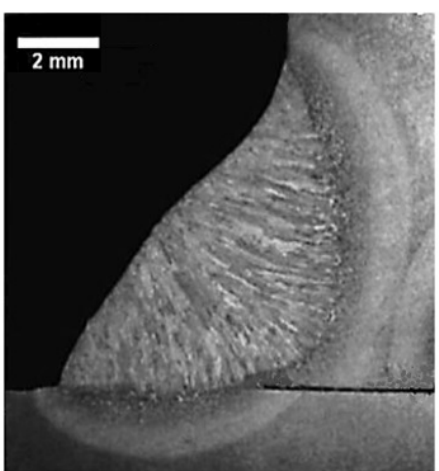

ME 30

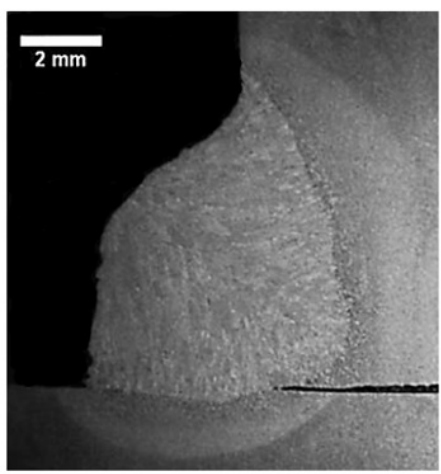

MA 30

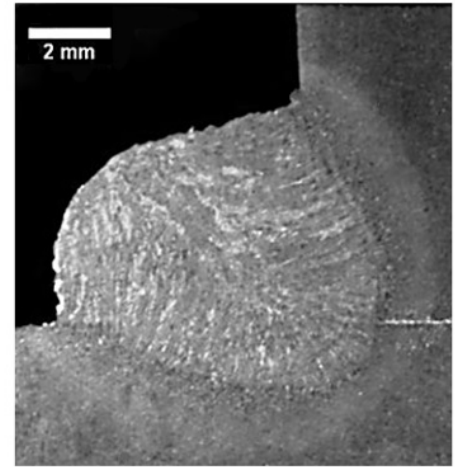

CA 45

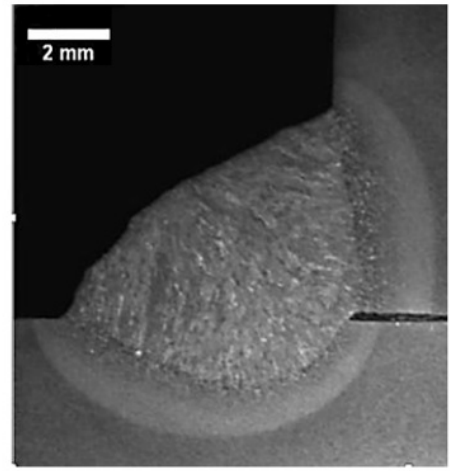

CE 45

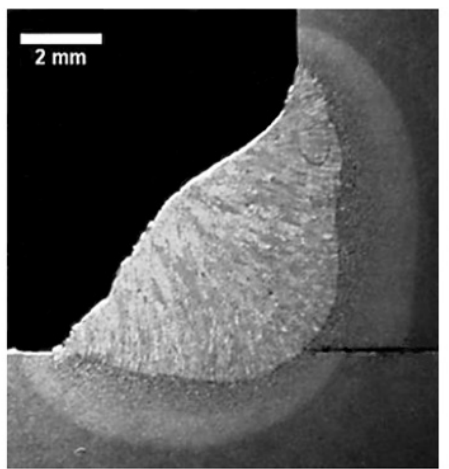

ME 45

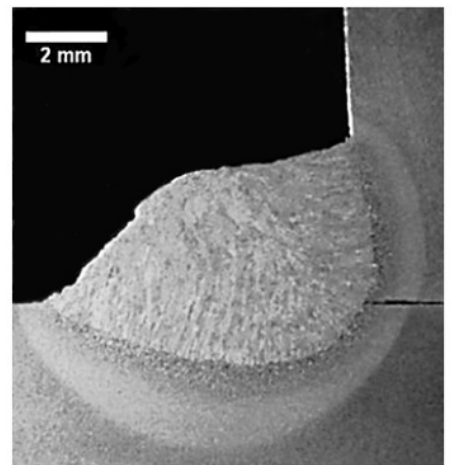

MA 45

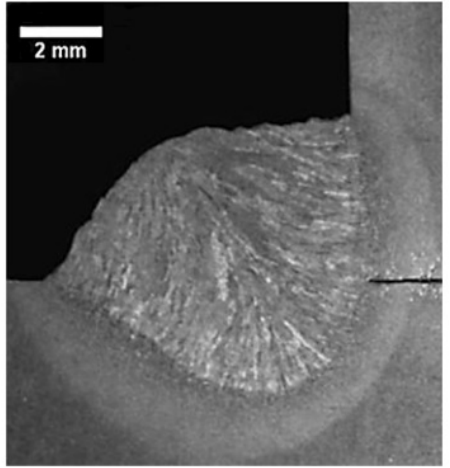

CA 60

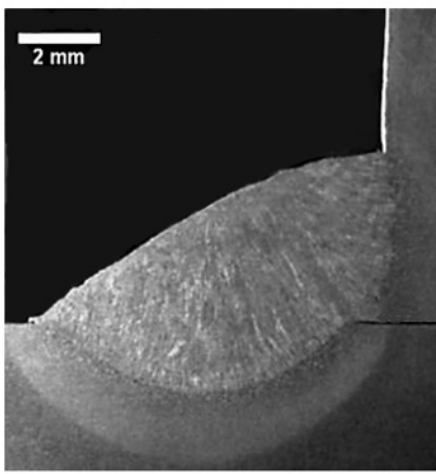

CE 60

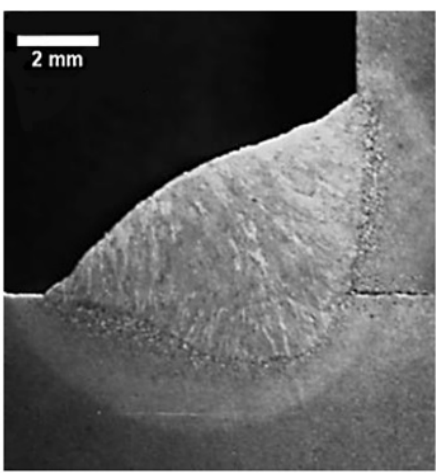

ME 60

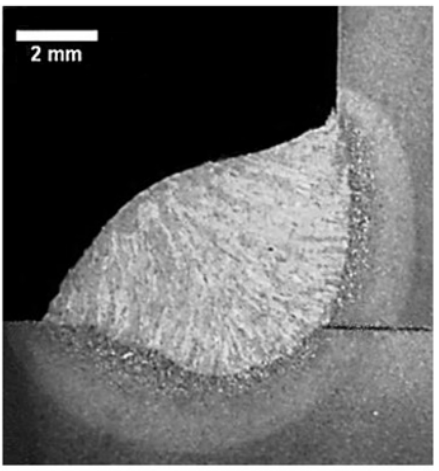

MA 60

Figura 9. Macrografías de las probetas soldadas bajo las distintas configuraciones. 


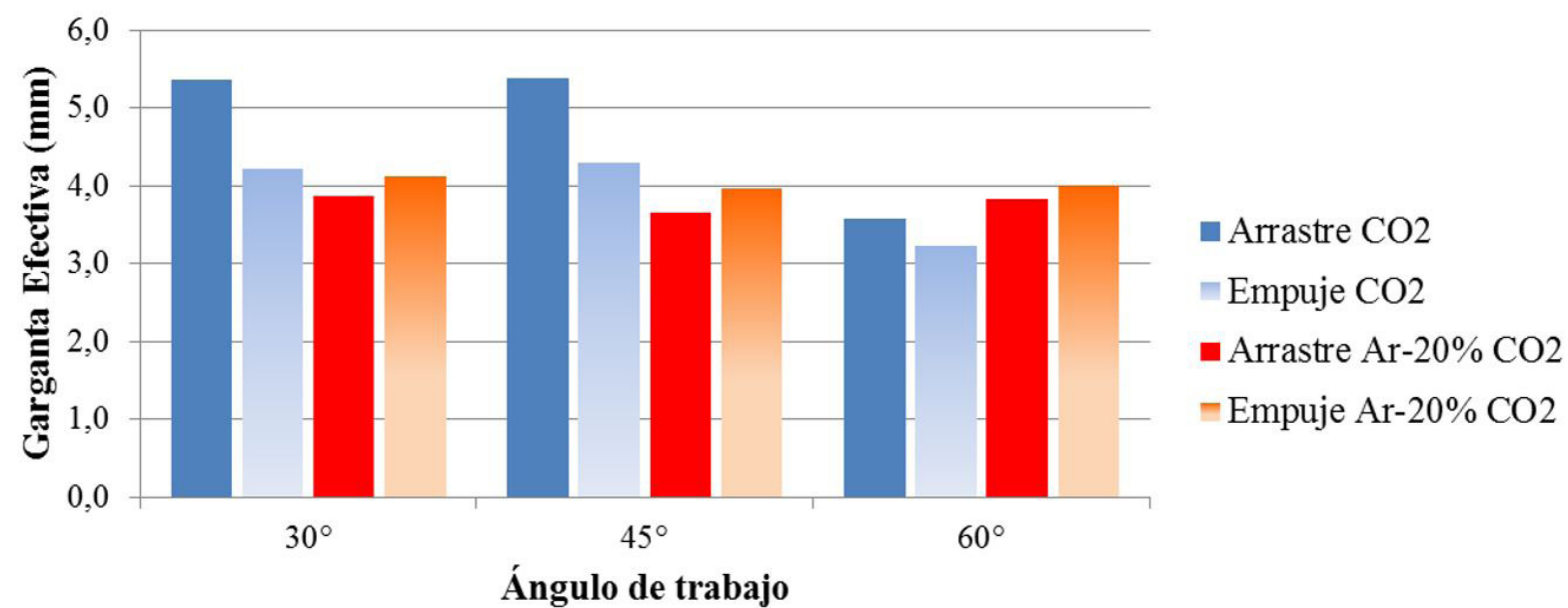

Figura 10. Efecto del gas de protección y el ángulo de desplazamiento sobre la penetración.

Tabla 4. Mediciones de las características geométricas de las probetas, en $\mathrm{mm}$.

\begin{tabular}{|c|c|c|c|c|c|c|c|c|c|c|c|c|}
\hline \multirow[t]{2}{*}{ Probeta } & \multicolumn{2}{|c|}{ Lados } & \multicolumn{2}{|c|}{ Penetración } & \multicolumn{2}{|c|}{ Garganta } & \multirow{2}{*}{$\begin{array}{l}\text { Sobre } \\
\text { espesor }\end{array}$} & \multirow{2}{*}{$\begin{array}{c}\text { Dilución } \\
\%\end{array}$} & \multicolumn{2}{|c|}{$\begin{array}{l}\text { Áng. de } \\
\text { mojado }\end{array}$} & \multicolumn{2}{|c|}{$\begin{array}{l}\text { Áng. de } \\
\text { fusión }\end{array}$} \\
\hline & $L_{1}$ & $L_{2}$ & $L_{1}$ & $L_{2}$ & $G_{t}$ & $\mathbf{G}_{\mathrm{e}}$ & & & $L_{1}$ & $\mathrm{~L}_{2}$ & $\mathrm{~L}_{1}$ & $L_{2}$ \\
\hline CA 30 & 6,5 & 5,8 & 1,5 & 1,8 & 4,4 & 5,4 & 1,1 & 37 & $50^{\circ}$ & $74^{\circ}$ & $17^{\circ}$ & $19^{\circ}$ \\
\hline CA 45 & 5,6 & 6,2 & 2,0 & 1,6 & 4,2 & 5,4 & 1,6 & 37 & $71^{\circ}$ & $87^{\circ}$ & $28^{\circ}$ & $24^{\circ}$ \\
\hline CA 60 & 3,8 & 6,8 & 0,6 & 2,6 & 3,3 & 3,6 & 1,3 & 41 & $81^{\circ}$ & $41^{\circ}$ & $39^{\circ}$ & $24^{\circ}$ \\
\hline CE 30 & 7,8 & 5,0 & 2,7 & 0,2 & 4,2 & 4,2 & 1,0 & 34 & $31^{\circ}$ & $74^{\circ}$ & $29^{\circ}$ & $13^{\circ}$ \\
\hline CE 45 & 5,3 & 6,5 & 0,8 & 1,6 & 4,0 & 4,3 & 0,9 & 30 & $50^{\circ}$ & $39^{\circ}$ & $25^{\circ}$ & $15^{\circ}$ \\
\hline CE 60 & 3,2 & 7,8 & 0,4 & 1,8 & 3,6 & 3,2 & 0,7 & 31 & $84^{\circ}$ & $39^{\circ}$ & $32^{\circ}$ & $14^{\circ}$ \\
\hline ME 30 & 7,3 & 5,1 & 1,4 & 0,1 & 4,6 & 4,1 & 0,3 & 28 & $37^{\circ}$ & $41^{\circ}$ & $25^{\circ}$ & $6^{\circ}$ \\
\hline ME 45 & 6,7 & 5,9 & 1,0 & 0,9 & 3,8 & 4,0 & 0,6 & 32 & $18^{\circ}$ & $49^{\circ}$ & $46^{\circ}$ & $20^{\circ}$ \\
\hline ME 60 & 4,9 & 7,7 & 0,4 & 1,8 & 4,1 & 4,0 & 0,6 & 27 & $58^{\circ}$ & $32^{\circ}$ & $21^{\circ}$ & $28^{\circ}$ \\
\hline MA 30 & 7,9 & 3,7 & 2,9 & 0,4 & 3,3 & 3,9 & 1,4 & 42 & $20^{\circ}$ & $79^{\circ}$ & $36^{\circ}$ & $9^{\circ}$ \\
\hline MA 45 & 4,0 & 7,6 & 0,8 & 1,6 & 3,4 & 3,7 & 1,0 & 37 & $65^{\circ}$ & $28^{\circ}$ & $35^{\circ}$ & $26^{\circ}$ \\
\hline MA 60 & 5,1 & 6,4 & 0,4 & 1,2 & 4,0 & 3,8 & 1,0 & 22 & $48^{\circ}$ & $66^{\circ}$ & $18^{\circ}$ & $14^{\circ}$ \\
\hline
\end{tabular}

$\mathrm{L}_{1}$ : lado $1 ; \mathrm{L}_{2}$ : lado $2 ; \mathrm{G}_{\mathrm{t}}$ : garganta teórica; $\mathrm{G}_{\mathrm{e}}$ : garganta efectiva.

la Tabla 4, y correspondieron a las probetas soldadas con 30 y $60^{\circ}$ de ángulo de trabajo por empuje, para la mezcla Ar- $20 \% \mathrm{CO}_{2}$ y el $100 \% \mathrm{CO}_{2}$ respectivamente.

Si la penetración más allá de la raíz puede ser lograda, el tamaño del lado o cateto puede ser reducido y la misma resistencia de la soldadura puede ser alcanzada. Esto reduce la cantidad requerida de metal de aporte y, si la penetración de la junta de filete puede ser hecha a la misma o más alta velocidad de soldadura, los costos de la soldadura pueden ser reducidos [17].

En todos los casos el sobre espesor estuvo por debajo de los $2 \mathrm{~mm}$ máximos permitido por la Especificación [14]. El mismo fue menor en la configuración por empuje para todas las condiciones de ángulos de trabajo, observándose una diferencia significativa lo cual resulta una consecuencia de la técnica empleada [4]. El mejor desempeño se obtuvo para la mezcla de gases y $30^{\circ}$ por empuje como se puede apreciar en la Figura 11.

Para similar ángulo de trabajo, la mayor dilución resultó para las probetas soldadas por arrastre, obteniéndose en general con el $\mathrm{CO}_{2}$, una mayor área fundida del metal base de acuerdo a los gráficos de la Figura 12 y en coincidencia a lo reportado por la bibliografía [4].

En general, los cordones resultaron levemente convexos, por ende el ángulo de mojado estuvo dentro de $45-75^{\circ}$. El único caso alejado al ideal fue para la probeta CA 45 donde la convexidad fue excesiva de acuerdo a la 


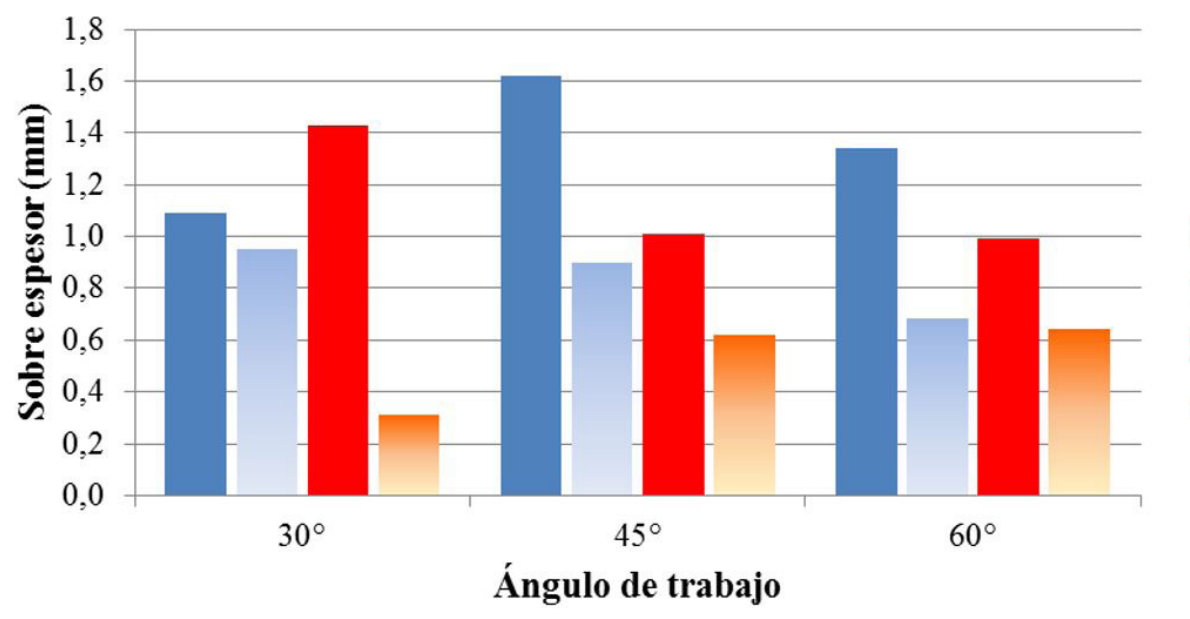

- Arrastre $\mathrm{CO} 2$

Empuje $\mathrm{CO} 2$

Arrastre $\mathrm{Ar}-20 \% \mathrm{CO} 2$

- Empuje Ar-20\% CO2

Figura 11. Efecto del ángulo de trabajo y desplazamiento sobre el sobre espesor.

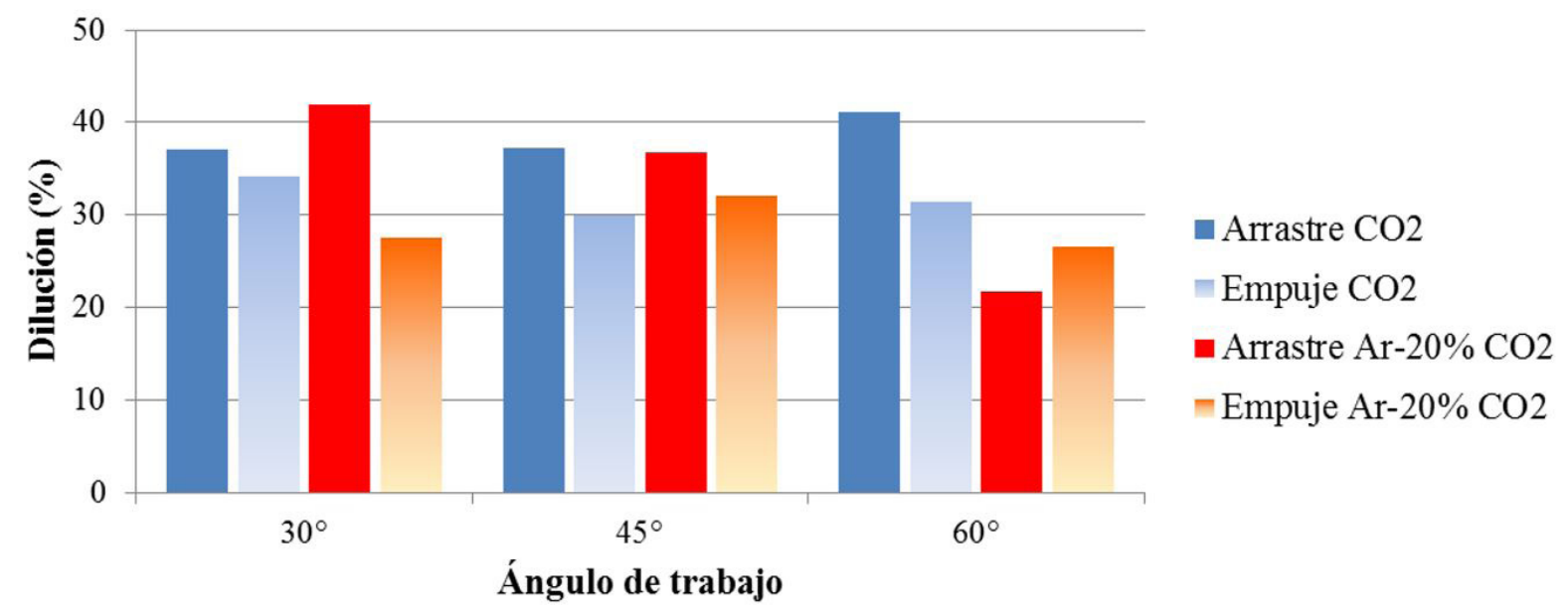

Figura 12. Efecto del gas de protección y el ángulo de desplazamiento sobre la dilución.

bibliografía [4]. Se observa también que, en promedio, las probetas soldadas con la mezcla de gases presentó un perfil más plano con un ángulo de mojado cercano a los $40-70^{\circ}$, mientras que con el $\mathrm{CO}_{2}$ este ángulo fue un poco mayor entre 50 a $80^{\circ}$.

En cuanto al ángulo de fusión y la penetración de los lados se observan para el $\mathrm{CO}_{2}$ valores mayores comparados con la mezcla de gases. La alta capacidad de transferencia de calor de gases como el $\mathrm{CO}_{2}$ favorece la eficiencia de fusión (zona fundida mayor), y la forma del cordón se asemeja a un plato hondo. Algunos autores acreditan una baja capacidad de transferencia de calor al Ar formando un cáliz de sus cordones [10].

A fin de valorar cuantitativamente aspectos cualitativos analizados, se desarrollaron índices para las diferentes mediciones realizadas que ponderan los resultados obtenidos para cada condición, respecto de lo conceptualmente deseado. Así se definieron los índices de lados $\left(I_{L}\right)$, de penetración de lados $\left(I_{\mathrm{PL}}\right)$, de penetración $\left(I_{\mathrm{P}}\right)$, de sobre espesor $\left(I_{S}\right)$, de dilución $\left(I_{D}\right)$, de ángulo de mojado $\left(I_{A M}\right)$ y de ángulo de fusión $\left(I_{A F}\right)$. A partir de los valores calculados para cada índice se asignó una ponderación de 0 a 3 , siendo 3 para la mejor condición y 0 para la peor de cada índice. En la Tabla 5 se muestran las definiciones de cada índice y los criterios de indexación de cada uno de los parámetros geométricos analizados.

En la Tabla 6 se muestran los resultados de la sumatoria de cada uno de los índices analizados, para cada probeta de estudio, dando como resultado un índice de aceptación geométrica $\left(\mathrm{I}_{\mathrm{A}}\right)$. El valor máximo de dicho índice es 21 . Se agregó una columna con las principales observaciones de los cordones analizados y en el caso de 
Tabla 5. Criterios de indexación de las características geométricas de las probetas.

\begin{tabular}{|c|c|c|c|c|c|c|c|c|c|c|c|c|c|}
\hline \multicolumn{2}{|c|}{$\begin{array}{c}I_{L} \\
1-\left[\left(L_{1}-L_{2}\right) /\right. \\
\left.x\left(L_{1}, L_{2}\right)\right]\end{array}$} & \multicolumn{2}{|c|}{$\begin{array}{c}I_{P L} \\
1-\left[\left(L_{1}-L_{2}\right) /\right. \\
\left.x\left(L_{1}, L_{2}\right)\right]\end{array}$} & \multicolumn{2}{|c|}{$\begin{array}{c}I_{p} \\
100 *\left(G_{e}-G_{t}\right) / G_{t} \\
(\%)\end{array}$} & \multicolumn{2}{|c|}{$\begin{array}{c}\mathrm{I}_{\mathrm{s}} \\
{\left[s /\left(\mathrm{G}_{\mathrm{t}}+\mathrm{s}\right)\right]^{*} 100} \\
(\%)\end{array}$} & \multicolumn{2}{|c|}{$\begin{array}{l}I_{D} \\
(\%)\end{array}$} & \multicolumn{2}{|c|}{$1-\left[\left(x\left(L_{1}, L_{2}\right)-50\right) / 50\right]$} & \multicolumn{2}{|c|}{$1-\left[\left(x\left(L_{1}, L_{2}\right)-30\right) / 30\right]$} \\
\hline $\begin{array}{l}>0,8 \\
<1,2 \\
\end{array}$ & 3 & $\begin{array}{l}>0,8 \\
<1,2\end{array}$ & 3 & $>15$ & 3 & $\begin{array}{l}>0 \\
<15 \\
\end{array}$ & 3 & $\begin{array}{l}>30 \\
<40 \\
\end{array}$ & 3 & $\begin{array}{l}>0,8 \\
<1,2\end{array}$ & 3 & $\begin{array}{l}>0,9 \\
<1,1 \\
\end{array}$ & 3 \\
\hline $\begin{array}{l}0,6 \text { a } 0,8 \\
1,2 \text { a } 1,4\end{array}$ & 2 & $\begin{array}{l}0 \text { a } 0,8 \\
1,2 \text { a } 2\end{array}$ & 2 & $\begin{array}{l}>5 \\
<15\end{array}$ & 2 & $\begin{array}{l}>15 \\
<25\end{array}$ & 2 & $\begin{array}{c}20 a \\
30 \\
40 a \\
50 \\
\end{array}$ & 2 & $\begin{array}{l}0,6 \text { a } 0,8 \\
1,2 \text { a } 1,4\end{array}$ & 2 & $\begin{array}{l}0,7 \text { a } 0,9 \\
1,1 \text { a } 1,3\end{array}$ & 2 \\
\hline $\begin{array}{l}0,3 \text { a } 0,6 \\
1,4 \text { a } 1,7\end{array}$ & 1 & $\begin{array}{l}-0,8 \text { a } 0 \\
2 \text { a } 2,8\end{array}$ & 1 & $\begin{array}{l}>0 \\
<5\end{array}$ & 1 & $\begin{array}{l}>25 \\
<35\end{array}$ & 1 & $\begin{array}{c}10 a \\
20 \\
50 a \\
60 \\
\end{array}$ & 1 & $\begin{array}{l}0,4 \text { a } 0,6 \\
1,4 \text { a } 1,6\end{array}$ & 1 & $\begin{array}{l}0,5 \text { a } 0,7 \\
1,3 \text { a } 1,5\end{array}$ & 1 \\
\hline $\begin{array}{l}<0,3 \\
>1,7 \\
\end{array}$ & 0 & $\begin{array}{l}<-0,8 \\
>2,8 \\
\end{array}$ & 0 & $<0$ & 0 & $>35$ & 0 & $\begin{array}{l}<20 \\
>60 \\
\end{array}$ & 0 & $\begin{array}{l}<0,4 \\
>1,6 \\
\end{array}$ & 0 & $\begin{array}{l}<0,5 \\
>1,5 \\
\end{array}$ & 0 \\
\hline
\end{tabular}

$\mathrm{I}_{\mathrm{L}}$ : índice de lados; $\mathrm{I}_{\mathrm{P}}$ : índice de penetración de lados; $\mathrm{I}_{\mathrm{P}}$ : índice de penetración; $\mathrm{I}_{\mathrm{S}}$ : índice de sobre espesor; $\mathrm{I}_{\mathrm{D}}$ : índice de dilución; $\mathrm{I}_{\mathrm{AM}}$ : índice de ángulo de mojado; $I_{A \mathrm{E}}$ : índice de ángulo de fusión; $x$ : promedio.

Tabla 6. Índices de las características geométricas de las probetas.

\begin{tabular}{|c|c|c|c|c|c|c|c|c|c|}
\hline Probeta & $I_{L}$ & $I_{P L}$ & $I_{p}$ & $I_{s}$ & $I_{D}$ & $I_{A M}$ & $\mathbf{I}_{\mathrm{AF}}$ & $I_{A}$ & Observaciones \\
\hline CA 30 & 3 & 2 & 3 & 2 & 3 & 3 & 1 & 17 & Perfil Deseable \\
\hline CA 45 & 3 & 2 & 3 & 1 & 3 & 1 & 2 & 15 & Excesiva convexidad \\
\hline CA 60 & 1 & 1 & 2 & 1 & 2 & 3 & 3 & 13 & $\begin{array}{l}\text { Aceptable. Lados desiguales. } \\
\text { Alta convexidad. Poca penetración } \mathrm{L}_{1}\end{array}$ \\
\hline CE 30 & 1 & 1 & 1 & 2 & 3 & 3 & 2 & 13 & $\begin{array}{l}\text { Aceptable. Poca garganta. } \\
\text { Insuficiente penetración } \mathrm{L}_{2}\end{array}$ \\
\hline CE 45 & 2 & 2 & 2 & 2 & 2 & 3 & 1 & 14 & Aceptable perfil \\
\hline CE 60 & 0 & 1 & 0 & 2 & 3 & 3 & 2 & 11 & $\begin{array}{l}\text { Insuficiente garganta y penetración } L_{1} \text {. Inadec- } \\
\text { uada penetración. }\end{array}$ \\
\hline ME 30 & 1 & 1 & 0 & 3 & 2 & 2 & 1 & 10 & $\begin{array}{l}\text { Insuficiente garganta y penetración } L_{2} \text {. Inadec- } \\
\text { uada penetración. }\end{array}$ \\
\hline ME 45 & 3 & 2 & 1 & 3 & 3 & 2 & 2 & 16 & Aceptable perfil \\
\hline ME 60 & 1 & 1 & 0 & 3 & 2 & 3 & 2 & 12 & Insuficiente garganta y penetración $\mathrm{L}_{1}$ \\
\hline MA 30 & 0 & 1 & 3 & 1 & 3 & 3 & 2 & 13 & $\begin{array}{l}\text { Aceptable perfil. Alta convexidad. } \\
\text { Insuficiente penetración } \mathrm{L}_{2}\end{array}$ \\
\hline MA 45 & 1 & 2 & 2 & 2 & 3 & 3 & 3 & 16 & $\begin{array}{l}\text { Aceptable perfil. Lados desiguales. } \\
\text { Alta convexidad }\end{array}$ \\
\hline MA 60 & 2 & 1 & 0 & 2 & 2 & 3 & 1 & 11 & Insuficiente garganta y penetración $\mathrm{L}_{1}$ \\
\hline
\end{tabular}

$\mathrm{I}_{\llcorner}$: índice de lados; $I_{\mathrm{PL}}$ : índice de penetración de lados; $\mathrm{I}_{\mathrm{P}}$ : índice de penetración; $\mathrm{I}_{\mathrm{S}}$ : índice de sobre espesor; $\mathrm{I}_{\mathrm{D}}$ : índice de dilución; $\mathrm{I}_{\mathrm{AM}}$ : índice de ángulo de mojado; $I_{A}$ : índice de ángulo de fusión; $I_{A}$ : índice de aceptabilidad.

cordones inaceptables según los requerimientos de la Especificación [14], el motivo del rechazo. Las condiciones sombreadas, tienen algún índice que implica el rechazo de la probeta. El principal índice que determinó el rechazo es el de penetración $\left(I_{p}\right)$.

Puede notarse que las condiciones CE 60, ME 30, ME 60 y MA 60 no cumplen con los requerimientos de la Especificación. En este sentido, se observa que para un ángulo de trabajo de $60^{\circ}$ se dificulta lograr la aceptación, siendo en general el problema la insuficiente penetración y tamaño de garganta. Asimismo, la mezcla de gases sería más sensible a estos aspectos. Por otro lado, las condiciones CA 30, CA 45, ME 45 y MA 45 presentaron los mejores resultados $\left(15<I_{A}<17\right)$, confirmando que la condición de $45^{\circ}$ de ángulo de trabajo con ángulo de arrastre es de las más favorables, independientemente del tipo de gas utilizado. 


\subsection{Propiedades mecánicas}

En la Figura 13 se muestran los valores promedio de microdureza correspondientes al MS para las distintas condiciones de soldadura analizadas. En todos los casos el MS resultó con una mayor dureza que el metal base. En general se obtuvieron mayores niveles de microdureza del MS para las probetas soldadas con la mezcla de gases. Este efecto puede ser atribuido a que el $\mathrm{CO}_{2}$ es altamente reactivo (oxidante) y tiende a ocasionar la pérdida del Mn y Si del MS, lo que afecta sus propiedades mecánicas y explica la disminución en la microdureza [10].
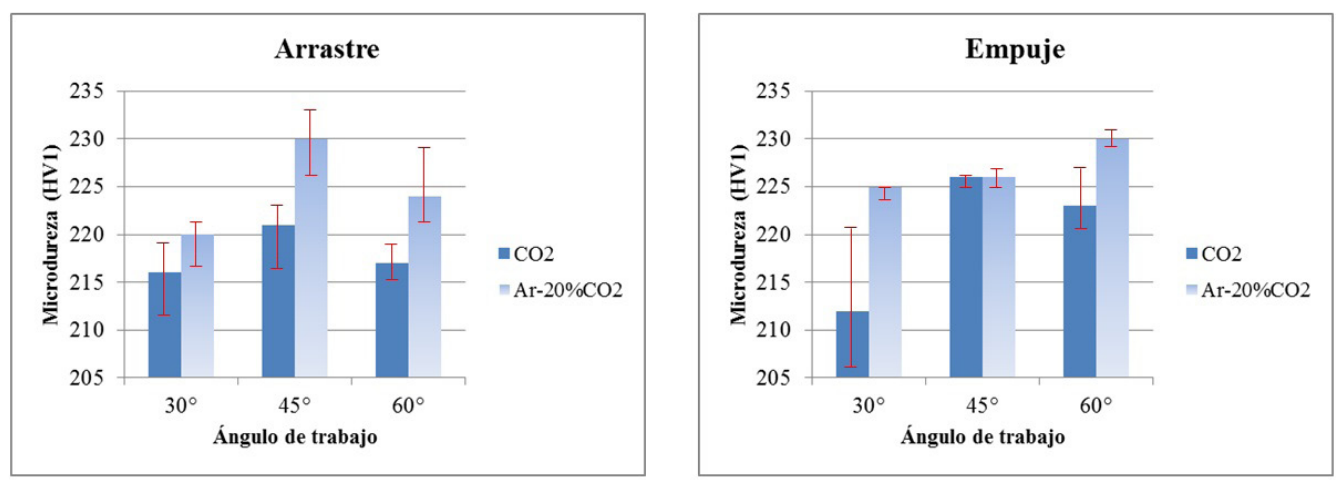

Figura 13. Microdureza del MS para las distintas condiciones de soldadura.

En la Tabla 7 se presentan los resultados del ensayo de rotura, expresado en carga por unidad de longitud de cordón $(\mathrm{N} / \mathrm{mm})$ de cada probeta para las juntas soldadas en diferentes condiciones. Esto se realiza a fin de independizarse de las diferencias que pudieran tener las mismas en esa dimensión. Tal como se mencionó en el punto 2.4 para cada condición se realizaron dos ensayos ( $\mathrm{A}$ y B) y luego se promediaron. Por otro lado, en la última columna de la tabla se muestra la región de la junta donde ocurrió la fractura y algunas observaciones respecto a la penetración en la raíz.

Tabla 7. Resultados del ensayo de rotura.

\begin{tabular}{|c|c|c|c|}
\hline Probeta & Carga & tura & Zona de Fractura y Observaciones \\
\hline \multirow{2}{*}{ CA 30} & A: 728 & & \multirow{2}{*}{ Las dos probetas no rompieron } \\
\hline & B: 710 & 719 & \\
\hline \multirow{2}{*}{ CA 45} & A: 544 & & \multirow{2}{*}{ La A comenzó la fractura por $L_{1}$ y la B por el MS } \\
\hline & B: 569 & ו & \\
\hline \multirow{2}{*}{ CA 60} & A: 375 & 358 & \multirow{2}{*}{ La A rompió por $\mathrm{L}_{1}$ y la $\mathrm{B}$ comenzó la fractura por $\mathrm{L}_{1}$} \\
\hline & B: 341 & 358 & \\
\hline \multirow{2}{*}{ CE 30} & A: 567 & 524 & \multirow{2}{*}{ La A comenzó la fractura por el MS y la B rompió por $\mathrm{L}_{2}$} \\
\hline & B: 483 & 524 & \\
\hline \multirow{2}{*}{ CE 45} & A: 436 & & \multirow{2}{*}{ La A comenzó la fractura por el MS y la B no rompió } \\
\hline & B: 481 & 458 & \\
\hline \multirow{2}{*}{ CE 60} & A: 233 & 203 & \multirow{2}{*}{ Las dos probetas comenzaron la fractura por $L_{1}$ y tienen falta de fusión en la raíz } \\
\hline & B: 173 & & \\
\hline \multirow{2}{*}{ ME 30} & A: 527 & 526 & \multirow{2}{*}{ Las dos probetas comenzaron la fractura por el MS } \\
\hline & B: 524 & $3<0$ & \\
\hline \multirow{2}{*}{ ME 45} & A: 588 & 601 & \multirow{2}{*}{ Las dos probetas comenzaron la fractura por el MS } \\
\hline & B: 614 & & \\
\hline \multirow{2}{*}{ ME 60} & A: 381 & 356 & \multirow{2}{*}{ Las dos probetas comenzaron la fractura por $\mathrm{L}_{1}$ y tienen falta de fusión en la raíz } \\
\hline & B: 331 & & \\
\hline \multirow{2}{*}{ MA 30} & A: 443 & 469 & \multirow{2}{*}{ La A comenzó la fractura por $L_{2}$ y la B por el MS } \\
\hline & B: 494 & & \\
\hline \multirow{2}{*}{ MA 45} & A: 471 & 472 & \multirow{2}{*}{ Las dos probetas comenzaron la fractura por $\mathrm{L}_{1}$} \\
\hline & B: 471 & $4 / 2$ & \\
\hline \multirow{2}{*}{ MA 60} & A: 440 & 439 & \multirow{2}{*}{ Las dos probetas comenzaron la fractura por $\mathrm{L}_{1}$} \\
\hline & B: 439 & 439 & \\
\hline
\end{tabular}


Efecto del Gas de Protección, Ángulos de Trabajo y Desplazamiento sobre las Características de Juntas de Filete Obtenidas por GMAW en un Acero Microaleado de Alta Resistencia

La Figura 14 muestra los tipos de fractura observados. La probeta CE 60, con la más baja resistencia, rompió a lo largo del lado 1, el cual tiene el menor cateto y penetración de ese lado. En cambio la probeta ME45, la segunda de mayor resistencia, rompió en el MS por el centro del cordón. En el caso de las probetas CA 30, con la más alta resistencia, no se produjo la fractura.

La Figura 15 muestra una comparación entre los resultados del ensayo de rotura para las probetas soldadas con $\mathrm{CO}_{2}$ y las soldadas con mezcla. Se observa para el $\mathrm{CO}_{2}$ un mejor desempeño para $30^{\circ}$ de ángulo de trabajo, tanto en arrastre como en empuje. En cambio, para la mezcla los mejores resultados fueron para $45^{\circ}$. En ambos casos los peores resultados se dieron para $60^{\circ}$. Si se compara el efecto de los gases sobre la resistencia mecánica se concluye que las probetas soldadas con mezcla presentan un mejor comportamiento, atribuido a la mayor resistencia del MS como se mencionó anteriormente para la microdureza.

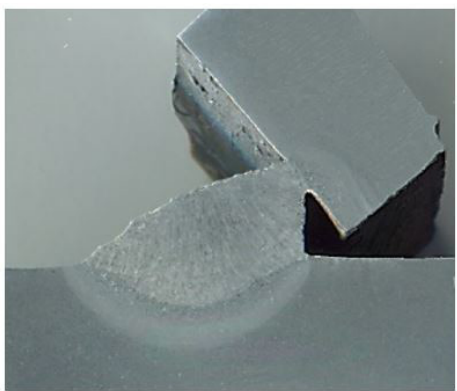

(a)

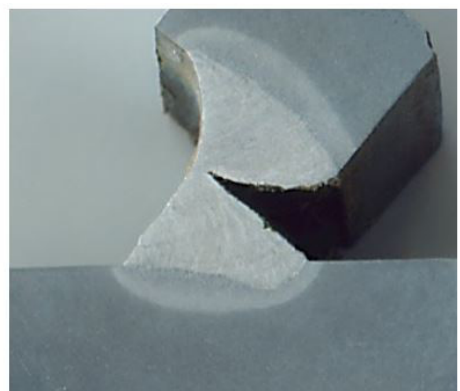

(b)

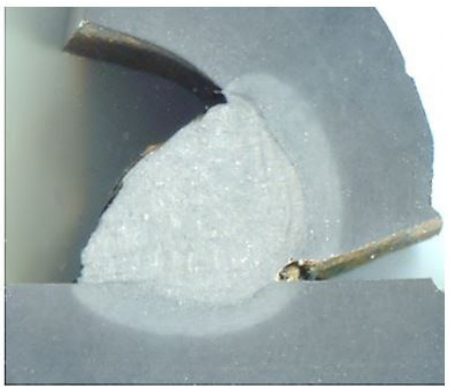

(c)

Figura 14. Macrofotografías de la zona de fractura de las probetas de rotura (a) CE 60; (b) ME 45; (c) CA 30.

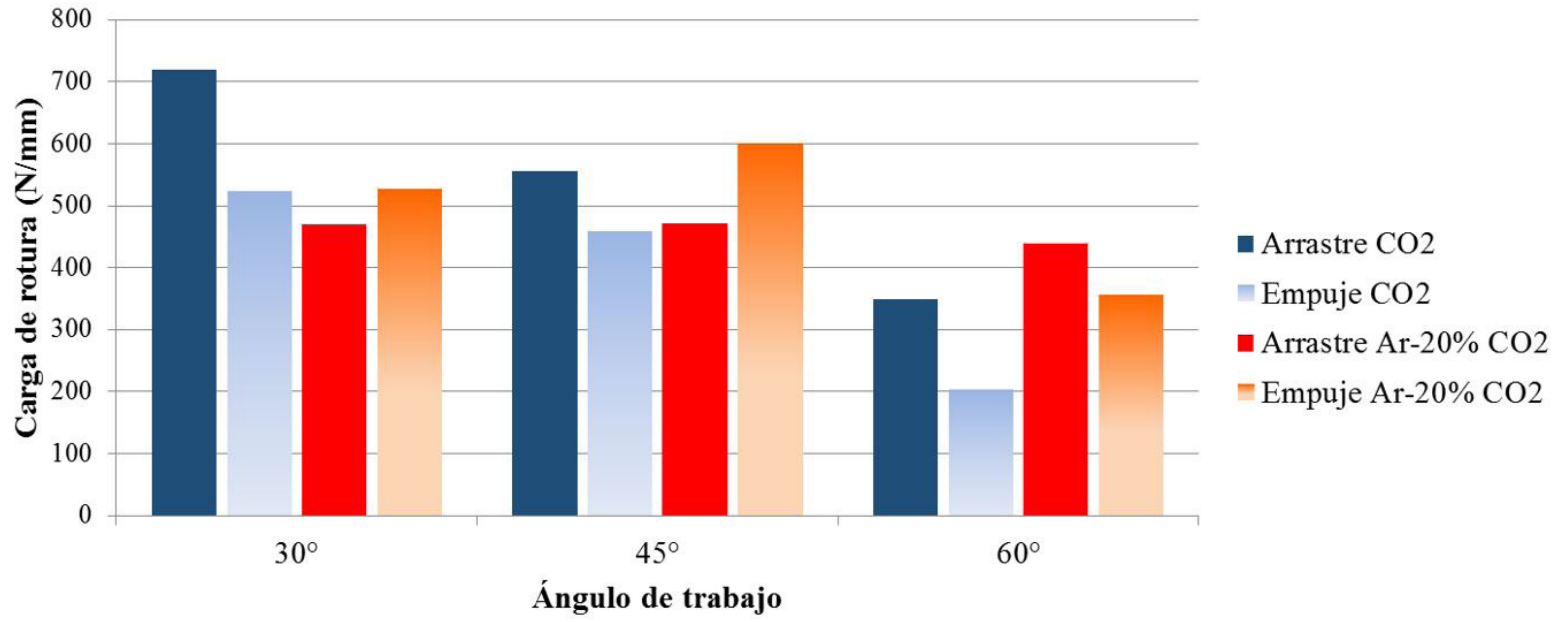

Figura 15. Efecto de las diferentes variables sobre la carga de rotura.

Los resultados obtenidos del ensayo de rotura fueron consistentes con lo determinado en el análisis dimensional, donde las condiciones CA 30, y ME 45 mostraron los mayores índices (16 y 17) y las mayores cargas de rotura (600-720). Esto pone de manifiesto la buena capacidad de predicción de los índices desarrollados así como su utilidad tecnológica.

\section{Conclusiones}

Al cambiar el gas de protección, el ángulo de trabajo y de desplazamiento, la morfología de los cordones se vio afectada sensiblemente alejándose de la condición ideal del ángulo de trabajo a $45^{\circ}$, tanto para arrastre como para empuje. 
Los mayores niveles de penetración y dilución correspondieron a las muestras soldadas con $\mathrm{CO}_{2}$ por arrastre. El sobre espesor fue menor para la condición por empuje, mostrando un mejor desempeño para la mezcla de gases, sumado a un perfil más adecuado del ángulo de mojado.

La microdureza aumentó levemente con el uso de la mezcla de gases, asociado a un menor nivel de oxidación de elementos de aleación. Las cargas de rotura variaron sensiblemente en función de las condiciones de soldadura.

Se determinó un índice de aceptación geométrico de la junta soldada, siendo la probeta soldada por arrastre con $\mathrm{CO}_{2}$ y a $30^{\circ}$ la que reunió el mejor desempeño. Asimismo, se logró correlacionar y verificar con los ensayos de rotura la capacidad de predicción de este índice.

Como conclusión general se puede destacar la importancia en la selección de los ángulos de trabajo y desplazamiento siendo la posición a $45^{\circ}$, tanto por arrastre como por empuje, la mejor opción para trabajar con la mezcla de gases. Sin embargo, si la accesibilidad a la junta es limitada lo recomendable es emplear el gas $\mathrm{CO}_{2}$ a $30 \circ 45^{\circ}$ y por arrastre. De esta forma, ajustando los parámetros de soldadura correctamente se podría alcanzar un nivel aceptable de calidad.

\section{Agradecimientos}

Los autores agradecen a los Laboratorios de Metalografía y de Ensayos de Materiales de la Escuela de Educación Secundaria Técnica N 1 "Antonio Bermejo" por la realización de los ensayos de rotura. También agradecen el apoyo financiero de la Universidad Tecnológica Nacional y de la UNNOBA.

\section{Referencias}

[1] Camilleri D, Molliconi P, Gray T. Computational methods and experimental validation of welding distortion models. Journal of Materials: Design and Applications. 2007;221(L4):235-249.

[2] Niebles E. Modelo de diseño y base de conocimiento en tecnologías de soldadura para el desarrollo de productos soldados. Cienthia y Technica. 2007;37(1):473-478.

[3] Mathers G. Job knowledge 91: design part 2 [página web]. Cambridge: TWI; 2016. [acceso el 4 marzo 2016]. Disponible www.twi-global.com

[4] Weman K, Lindén G. MIG welding guide. Cambridge: Woodhead Publishing; 2006.

[5] Jeffus L. Metal fabrication technology for agriculture. 2nd ed. United States of America: Delmar Cengage Learning; 2011.

[6] Morrett B. How to improve your welds: helpful hints for GMAW. Practical Welding Today. 1999.

[7] American Welding Society. Welding Handbook: welding processes: part 1. 9th ed. Miami: AWS; 2004. (vol. 2).

[8] ASM International. ASM Handbook: welding, brazing, and soldering. Materials Park: ASM; 1993. (vol. 6).

[9] Ebrahimnia M, Goodarzi M, Nouri M, Sheikhi M. Study of the effect of shielding gas composition on the mechanical weld properties of steel ST 37-2 in gas metal arc welding. Materials
\& Design. 2009;30(9):3891-3895. http://dx.doi.org/10.1016/j. matdes.2009.03.031.

[10] Scotti A, Ponomarev V. Soldagem MIG/MAG: melhor entendimento, melhor desempenho. São Paulo: Artliber; 2008.

[11] DeArdo AJ, Hua MJ, Cho KG, Garcia Cl. On strength of microalloyed steels: an interpretive review. Materials Science and Technology. 2009;25(9):1074-1082. http://dx.doi. org/10.1179/174328409X455233.

[12] Funderburk RS. Selecting filler metals: matching strength criteria. Welding Innovation. 1999;16(2):1-3.

[13] American Society for Testing and Materials. ASTM E8-04: standard test methods for tension testing of metallic materials. West Conshohocken: ASTM; 2004.

[14] American Welding Society. ANSI/AWS D14.3/D14.3M:2010: specification for welding earthmoving, construction, and agricultural equipment. Miami: AWS; 2010.

[15] American Welding Society. AWS A5.28/A5.28M:2005: specification for low-alloy steel electrodes and rods for gas shielded arc welding. Miami: AWS; 2005.

[16] Weman K. Welding Processes Handbook. 2nd ed. Cambridge: Woodhead Publishing.

[17] Miller DK. Consider penetration when determining fillet weld size. Welding Innovation. 1998;15(1):1-3. 